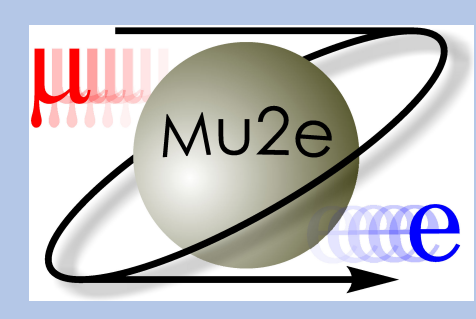

\title{
Mu2e Calorimeter Clustering Studies
}

\author{
Emma Castiglia \\ with Giani Pezzullo and Sarah Demers \\ Yale University
}

New Perspectives 2018 


\section{Mu2e Calorimeter Overview}

- Signal: $105 \mathrm{MeV}$ Conversion Electron (CE) without neutrinos

- Requirements of Calorimeter:

- Energy Resolution: 10\%

- Timing Resolution: 1ns

- Position Resolution: $1 \mathrm{~cm}$

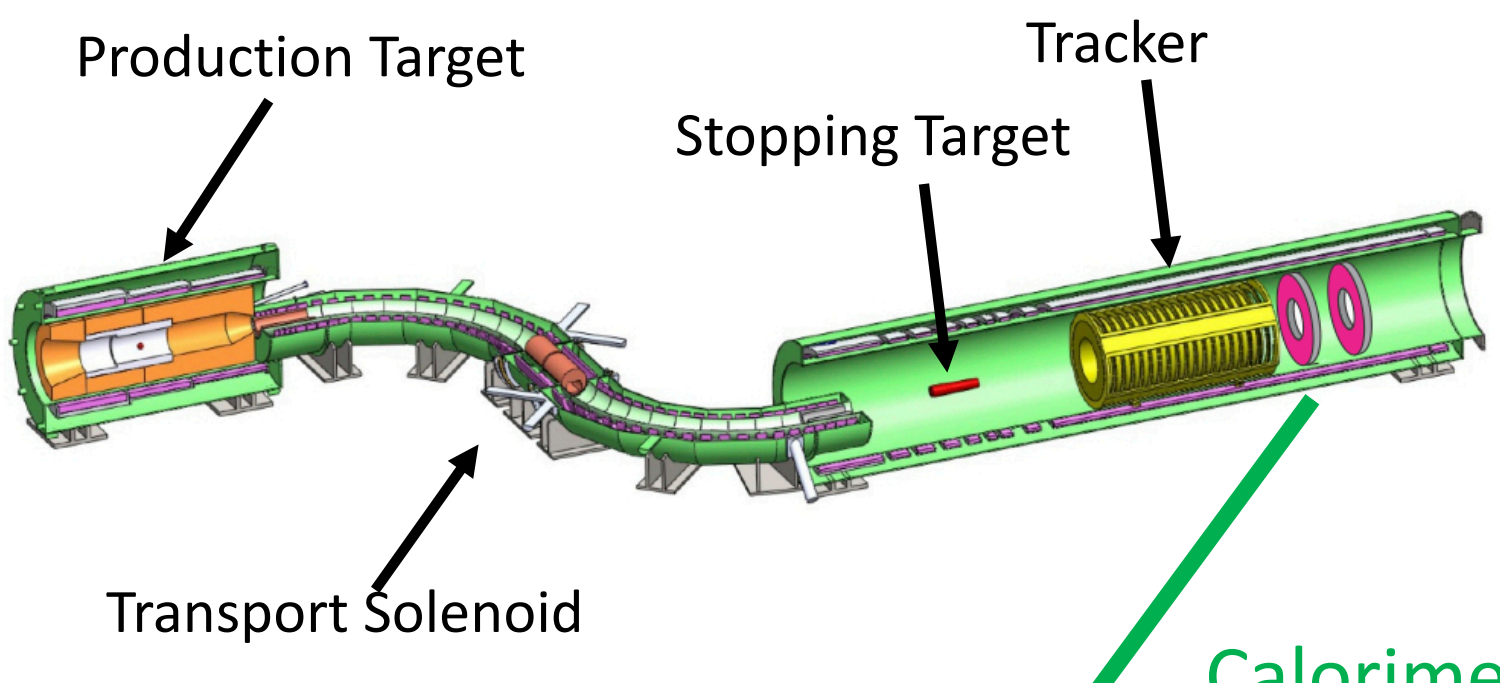

- Calorimeter Role:

- Particle Identification - reject bkg

- Cosmic Ray Muon

- Needs to be a trigger for data storage!

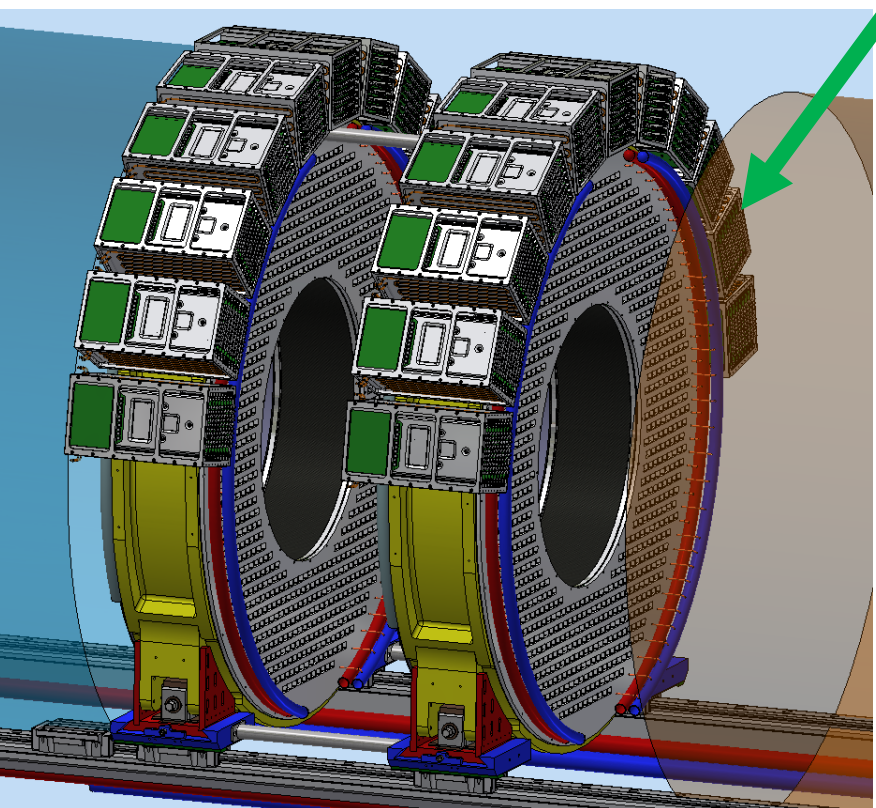


- Two annular disks of undoped CsI Crystals

- Dimensions: $3.4 \times 3.4 \times 20 \mathrm{~cm}^{3}$

- Radiation length of $2.1 \mathrm{~cm}$ ( 10 lengths in crystal)

- Total of 1356 crystals

- Crystals are read out by $2 \times 3$ of $6 \times 6 \mathrm{~mm}^{2}$ UV-extended SiPM

- Signal digitized at $200 \mathrm{MHz}$

- Measured performance at test beam (100 MeV)

- 100ps is time resolution

- $5 \%$ is energy resolution
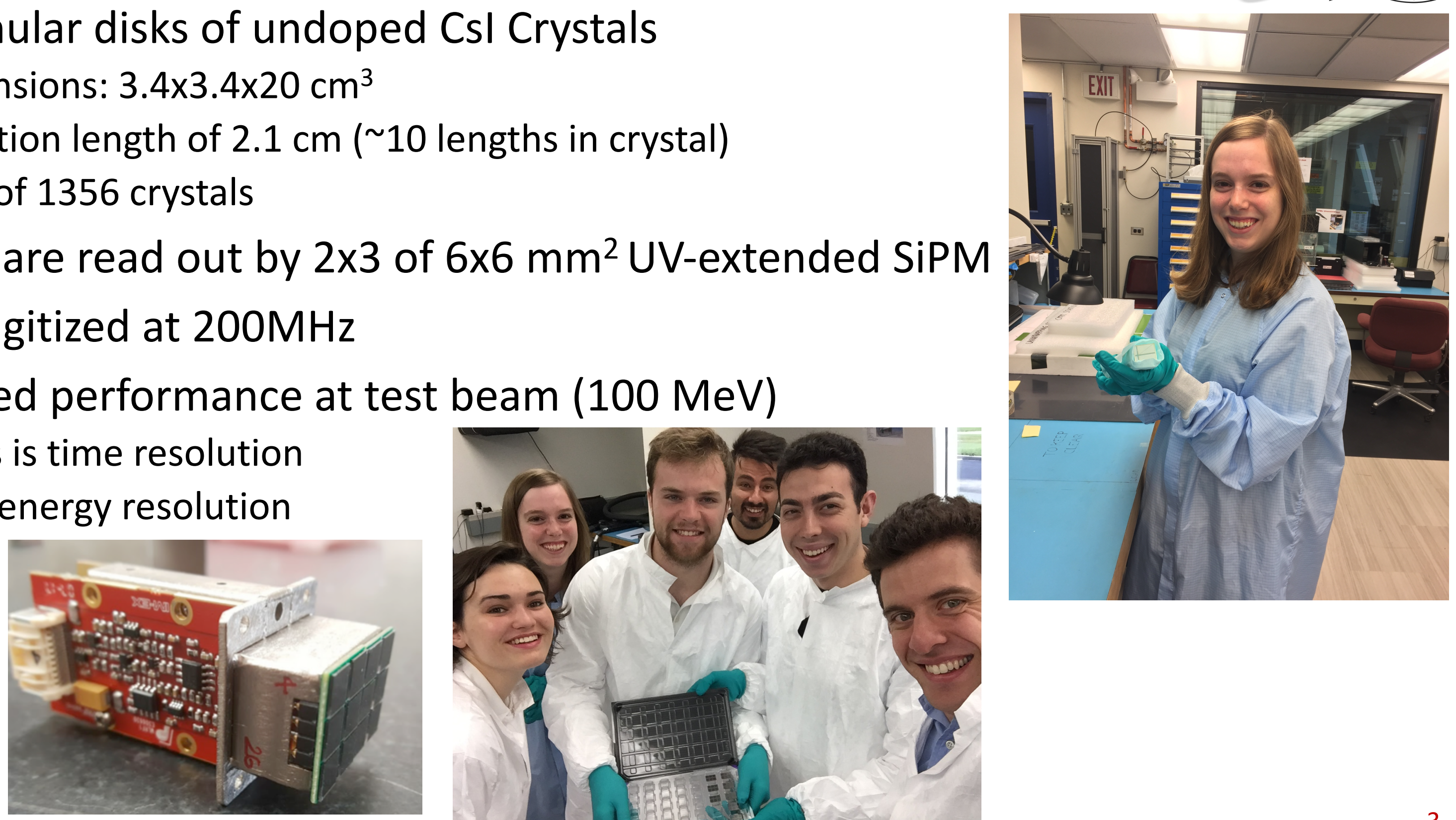


\section{Mu2e Calorimeter Clustering}

How it works?

- Sort crystal hits by energy deposited and then group adjacent ones

- 2 algorithms that do this differently

- Full (proto): Slower but more Accurate - use in offline reconstruction

- Fast: Quicker and Simple-could be trigger if runs in real time

- If able to identify potential signal events, can trigger on those events
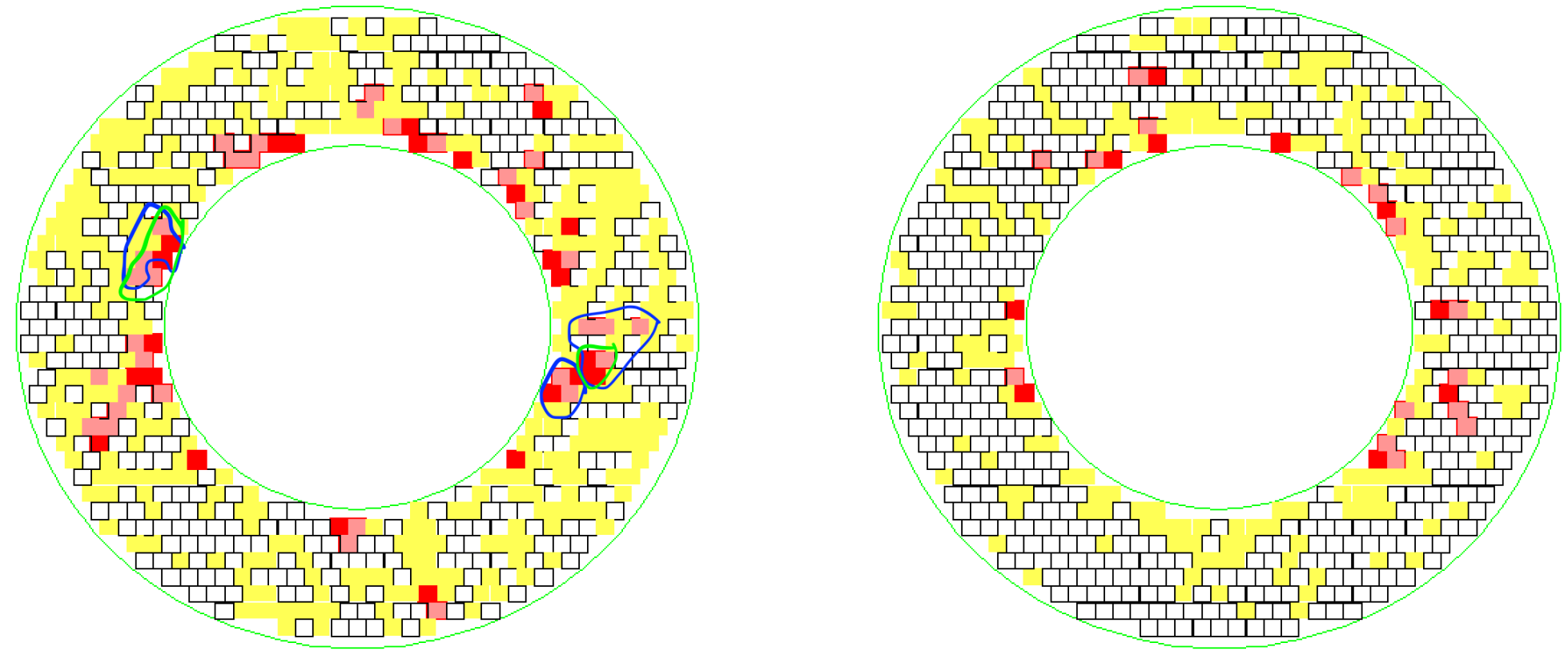


\section{Steps:}

1. Take highest energy crystal - seed with $>10 \mathrm{MeV}$

2. Look at neighboring crystals over energy threshold and group - green

3. Remove crystals that are clustered together from list of crystals

4. Start process over with next highest energy crystal

\section{Modification:}

Include next, next to next neighbors, etc Adds time:

1 ring -6 crystals

2 rings -12 crystals

3 rings -18 crystals

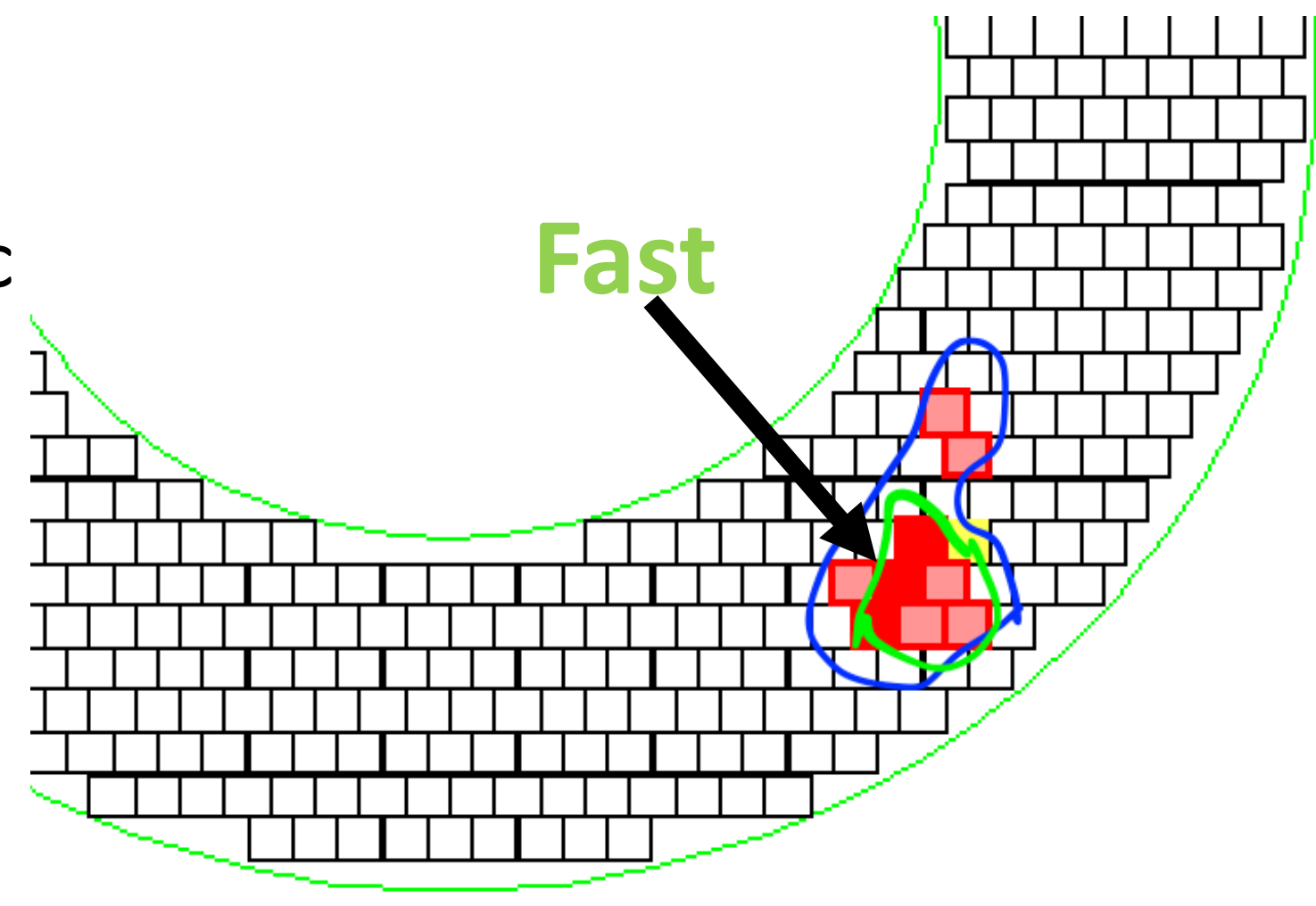




\section{Steps:}

1. Take highest energy crystal $\rightarrow>$ seed with $>10 \mathrm{MeV}$

2. Look at neighboring crystals over energy threshold and group

3. Look at non-adjacent crystals with large energy deposits - blue $\rightarrow$ could be deposited by photons emitted (within speed of light)

3a. Sometimes end up grouping two smaller clusters into one larger cluster

4. Remove crystals that are clustered together from list of crystals

5. Start process over with next highest energy crystal

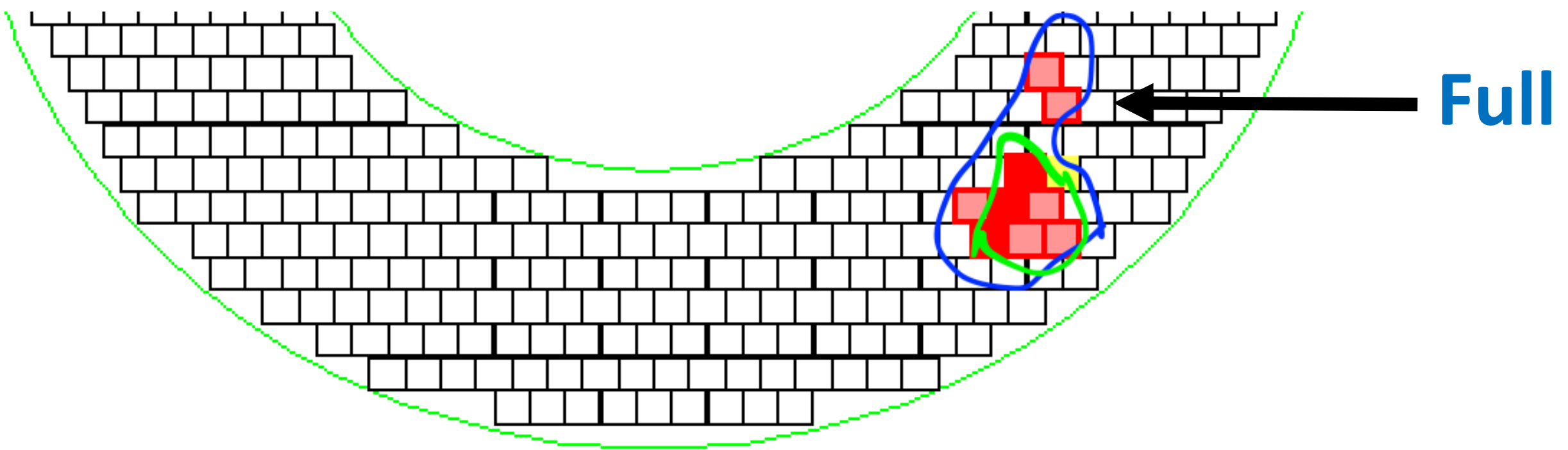


Goals for Clustering Studies

GOAL 1

Compare Fast and Full Algorithms for accuracy and timing performance

GOAL 2

Find discriminating variables for improving CE selection from background 

identifying conversion electrons (CE)?

Use information from the Virtual Detector

- G4 sensitive detector right before the calorimeter that stores the information of particles passing through it without affecting them

- Can get energy, radial position, etc. about the incoming CE - Truth - has conversion electron events that may miss the calorimeter 
Virtual Detector - Acceptance

Acceptance: 95\%

Leakage: Sharp Edge - detector is $20 \mathrm{~cm}$ deep and $30 \mathrm{~cm}$ wide

->shower loss due to depth/reflection

Energy resolution:

Full: $6.4 \%$

Fast: $8.6 \%$

FWHM:

Full: $6.2 \mathrm{MeV}$

Fast: $8.2 \mathrm{MeV}$

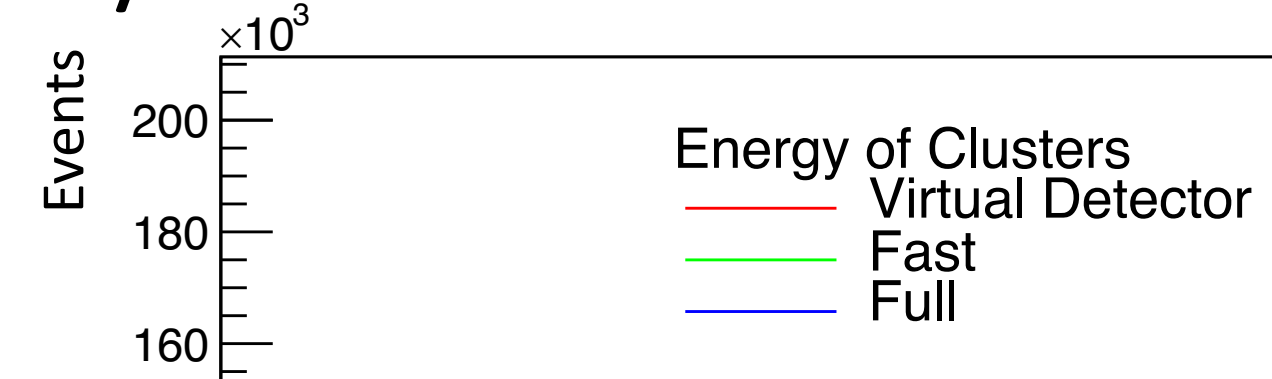




\section{Timing Comparison}

Have a few ms per event within which to trigger on or reject

\section{Calo Cluster Fast:}

- $\quad$.2ms per event

- Includes fast processing of digitized hits

\section{Full Clustering:}

- Algorithm takes $2.3 \mathrm{~ms}$ per event

- Needs Template Fit: $25 \mathrm{~ms}$ per event

- Complete time for each event is $>27 \mathrm{~ms}$

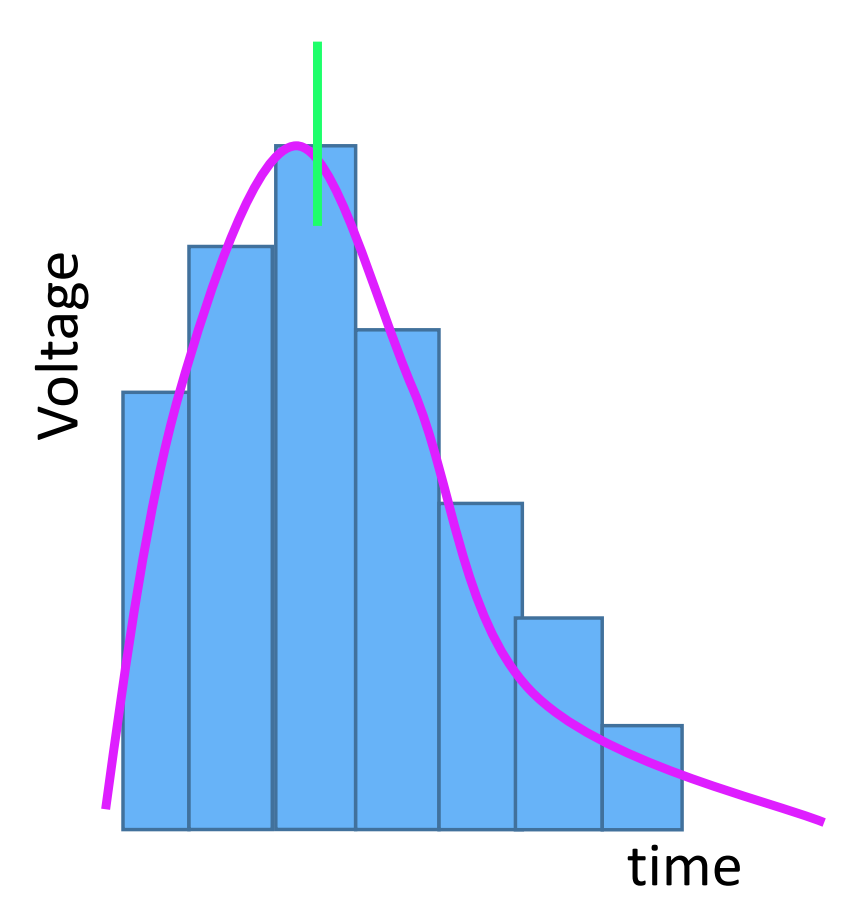

$=>$ Fast Algorithm is more robust - could use as trigger 
Energy versus Cluster Size - Fast

Distribution of Cluster Energy versus Cluster Size Fast

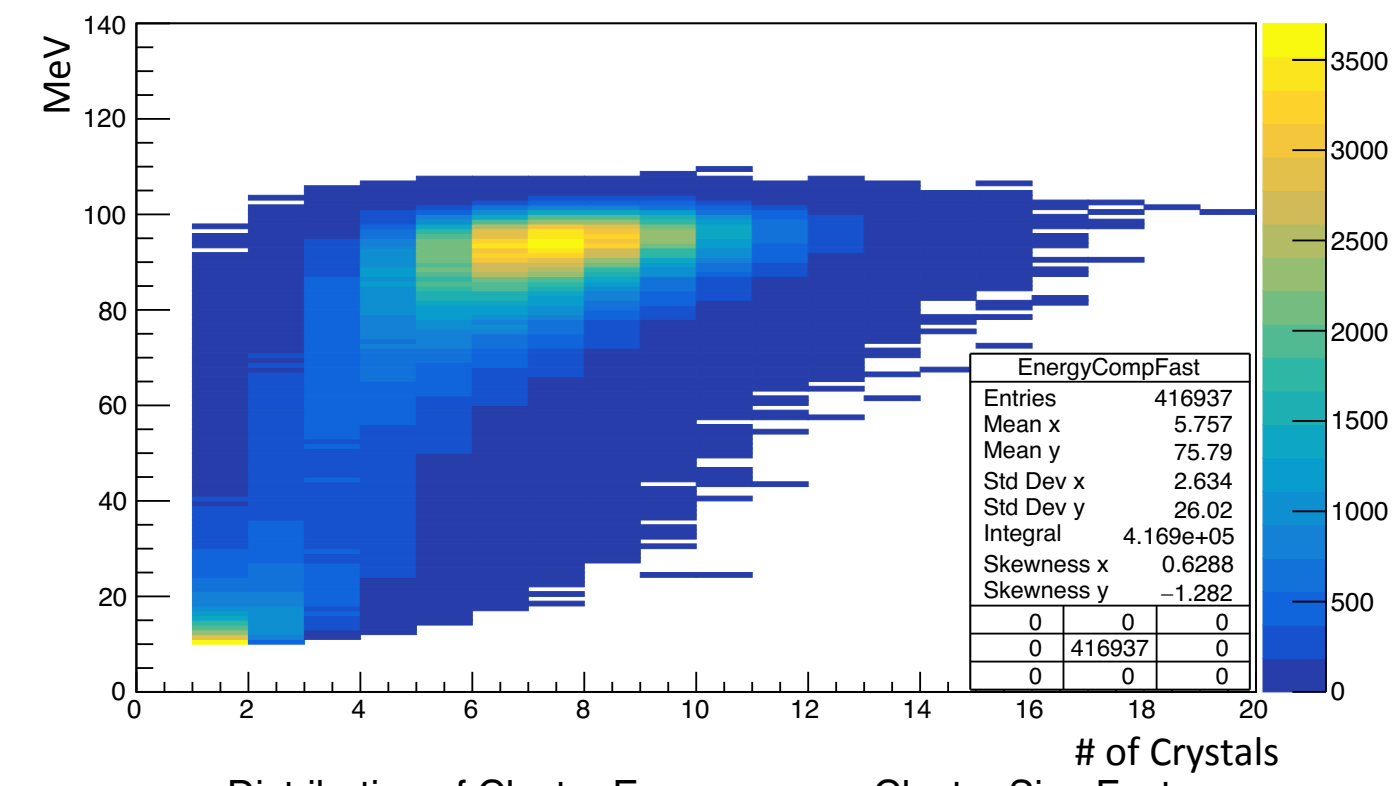

Distribution of Cluster Energy versus Cluster Size Fast

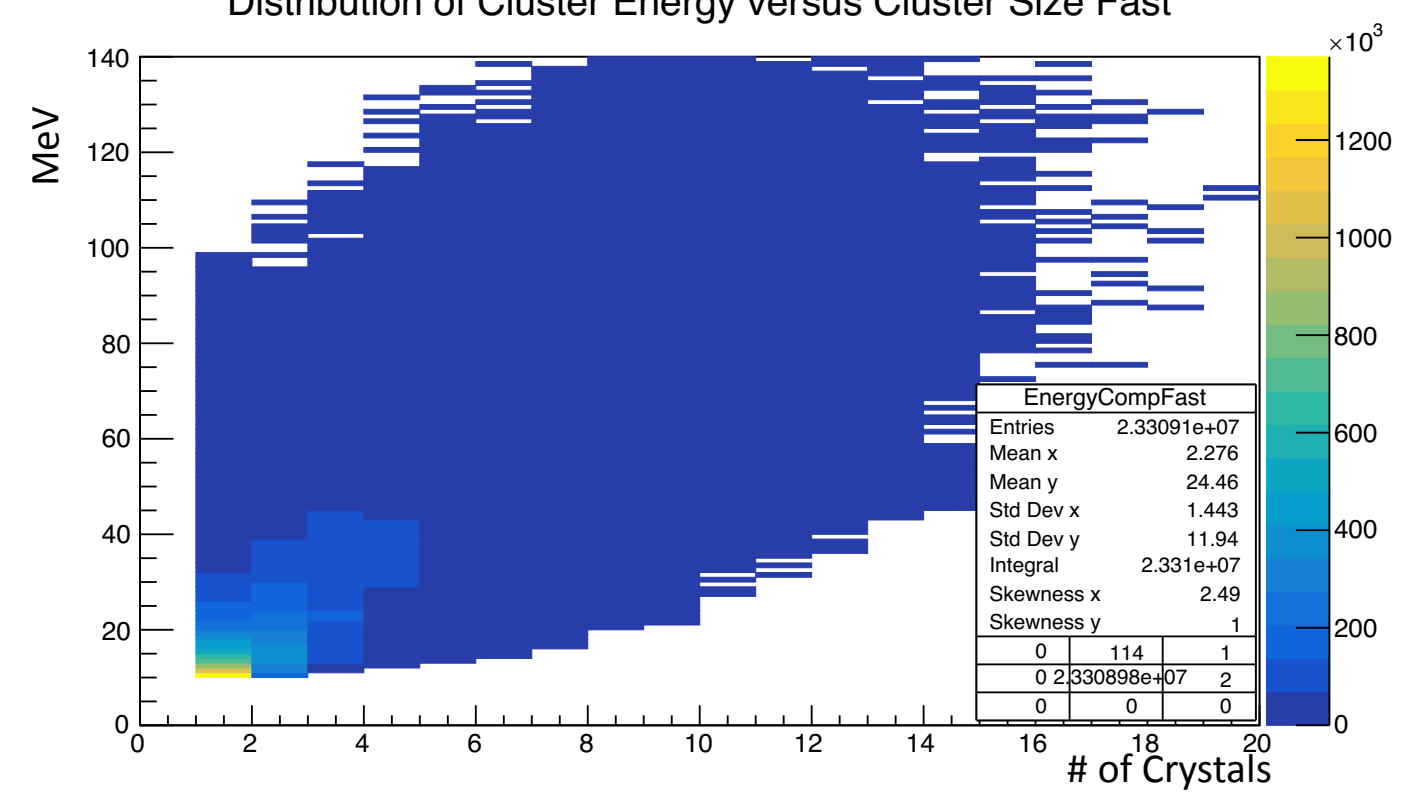

\section{CE Only:}

Peak at $80-100 \mathrm{MeV}$ and 6-9 crystals

\section{Background Only:}

Peak at $10 \mathrm{MeV}$ and 1 crystal
Calo Disk

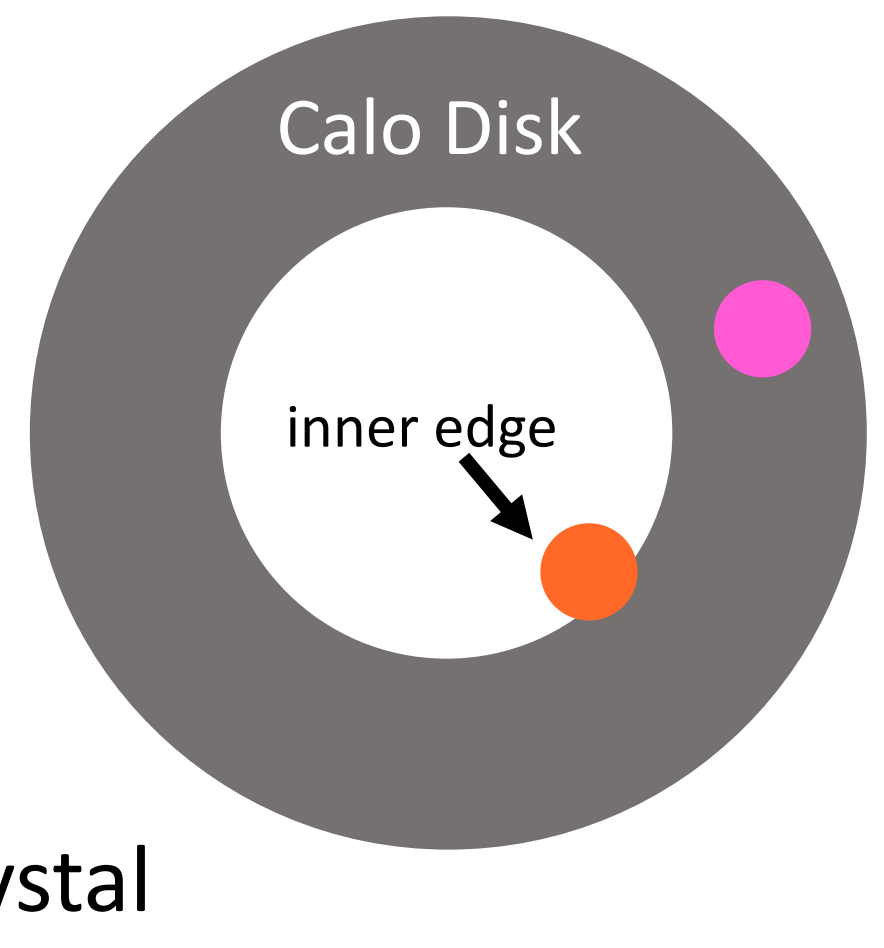




\section{ENERGY}

Drop off at $\sim 50 \mathrm{MeV}$

Distinctive background shape

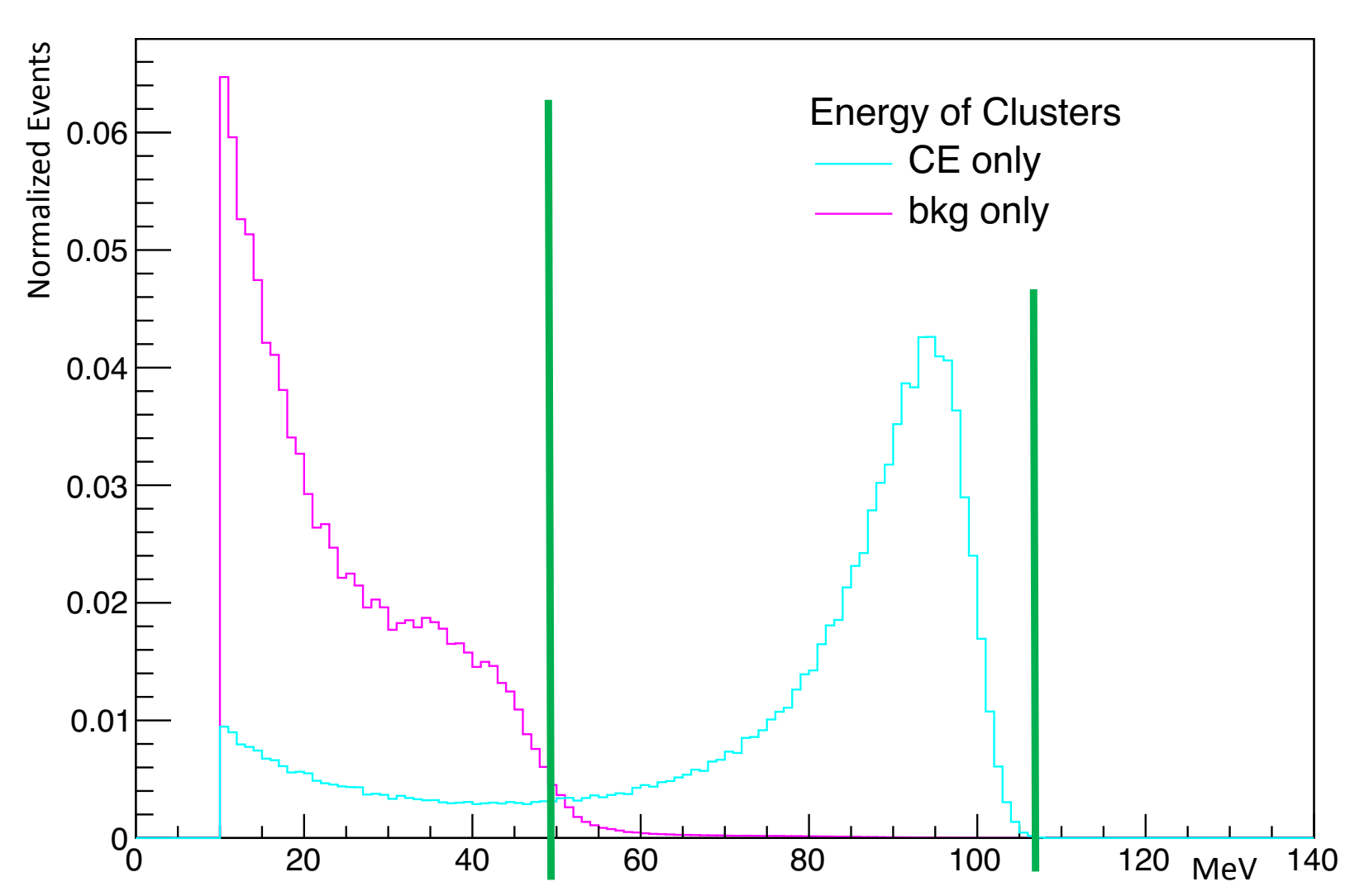

\section{RADIAL POSITION}

Drop off at $410 \mathrm{~mm}$

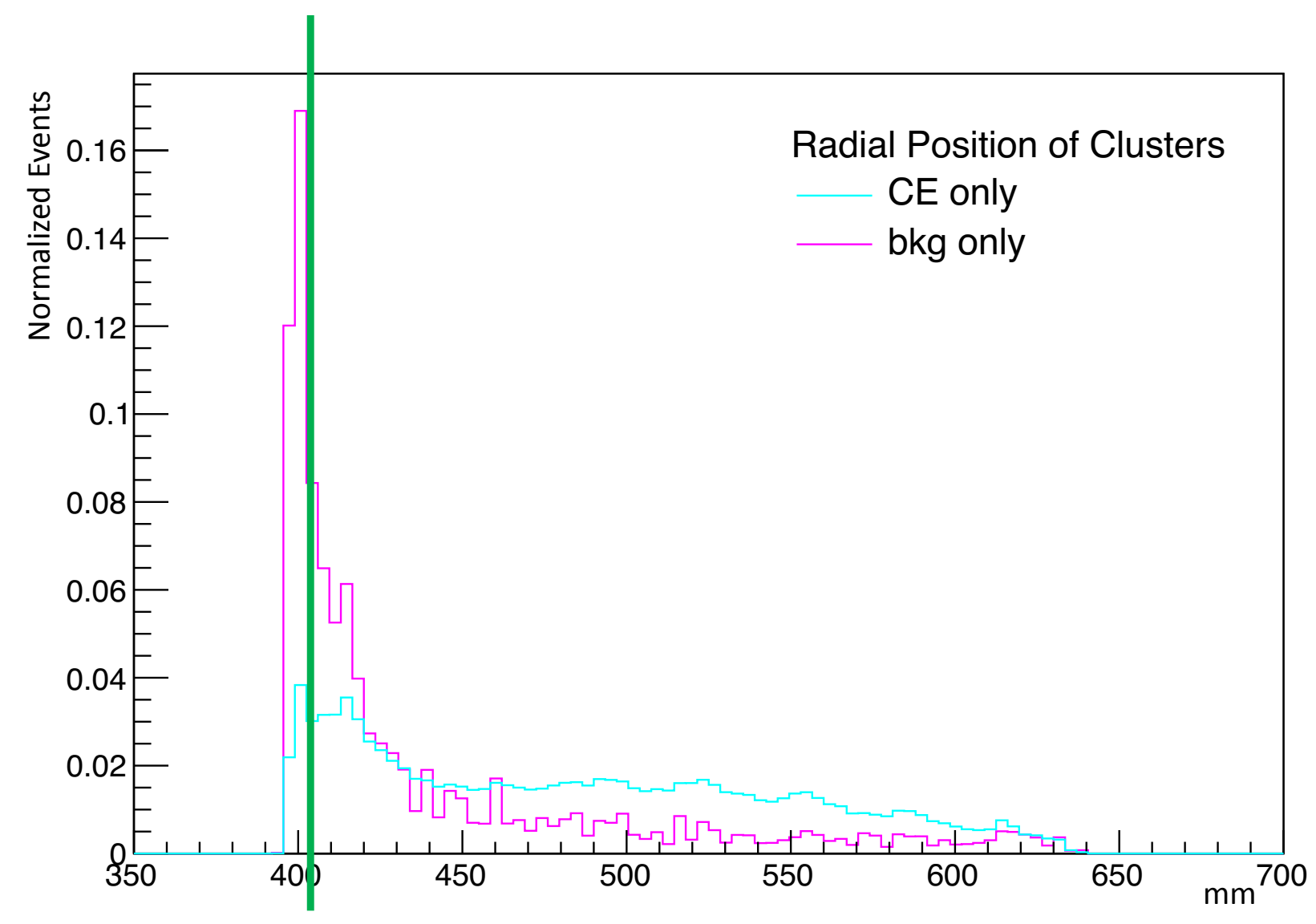


Comparing CE and bkg only - Fast RATIO OF SEED TO CLUSTER ENERGY

Background has more clusters with only 1 crystal

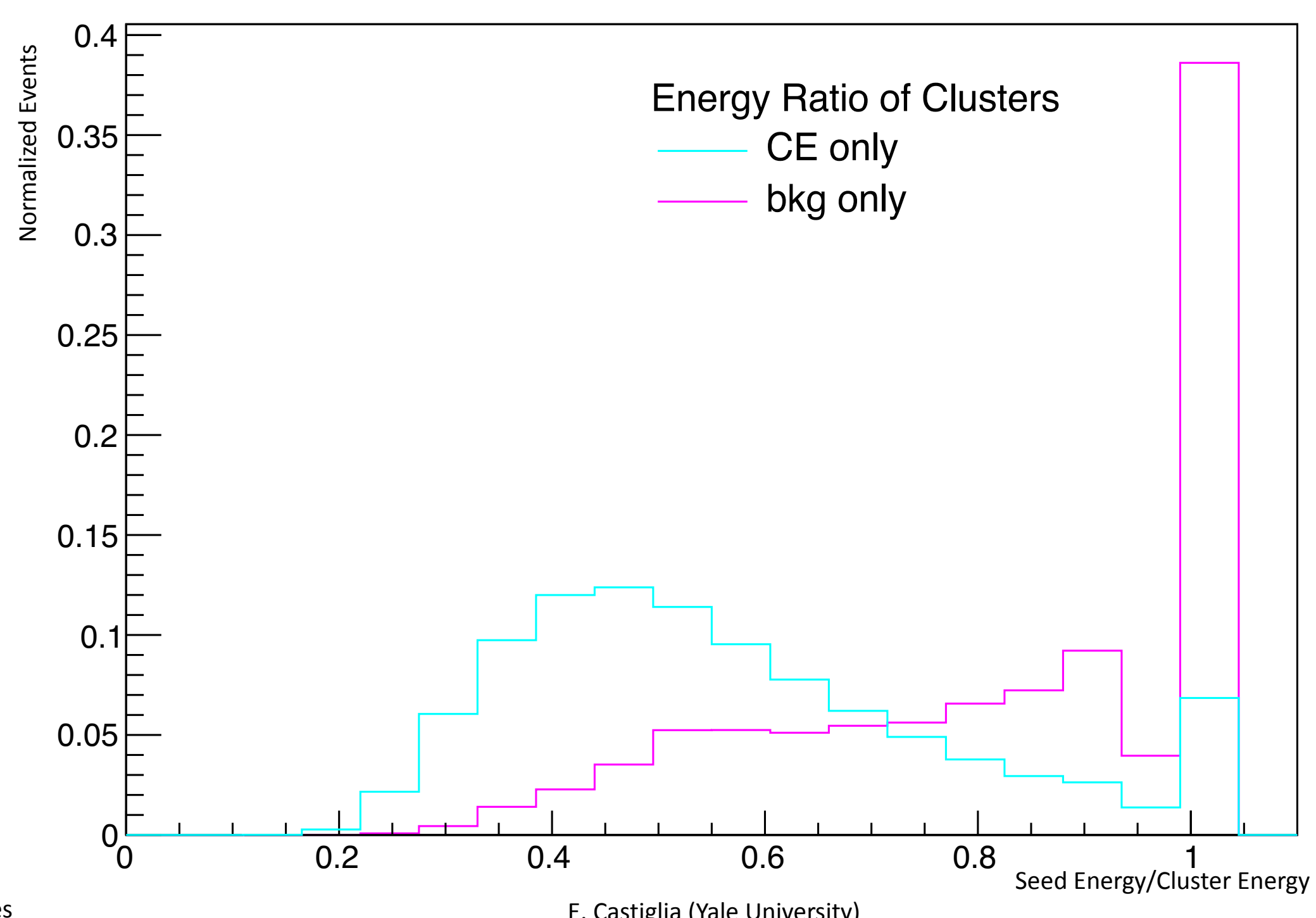




\section{Conclusion and Next Steps}

\section{Results so far:}

- Fast is quick and performs well enough to be used during triggering

- Energy resolution of Full could be improved in Offline

- Variables that could be used to differentiate:

- Energy and Size

- Radial Distance

- Ratio of Seed Energy to Cluster Energy

\section{Next Steps:}

- Look at adding more rings of neighbors to Fast

- Change minimum energy cutoff for clusters - currently $10 \mathrm{MeV}$ 
Questions?

0.0

\section{Thanks for listening!}


Backup Slides 


\section{FWHM Fits}

Cluster Energy in Fast
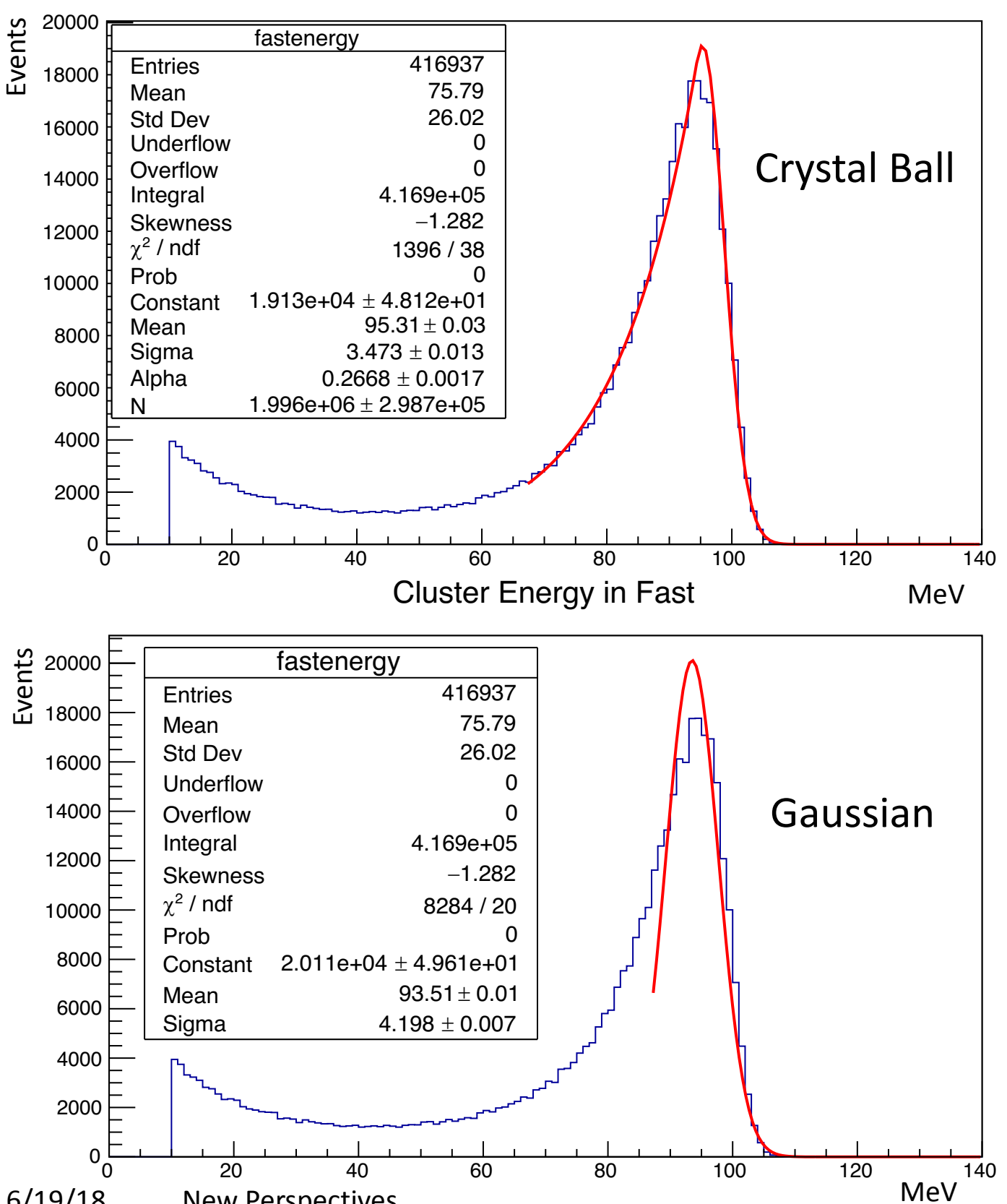

Cluster Energy in Proto
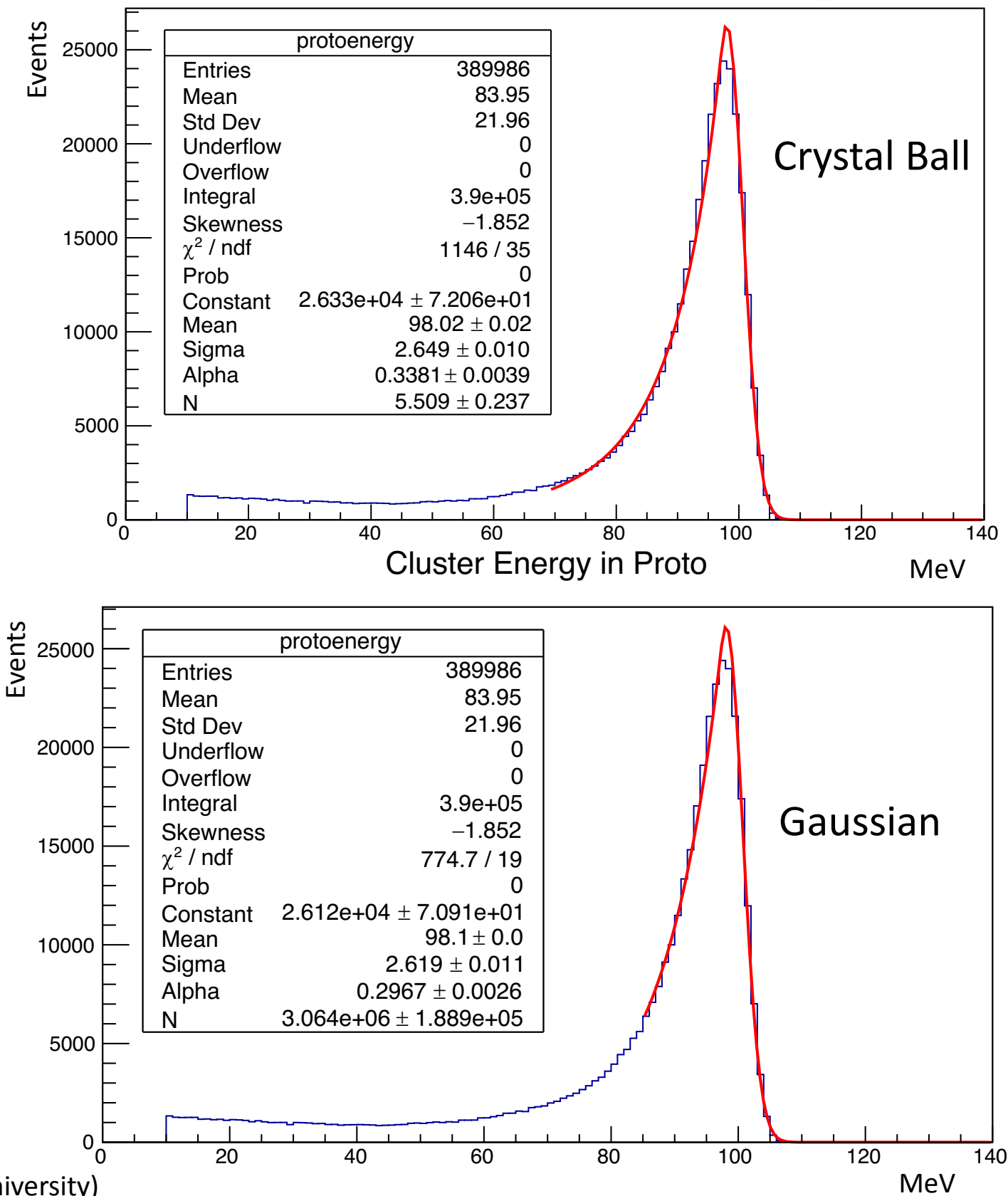


\section{MC comparison of Recon with VD}

- Reconstructed Clusters miss energy as compared to the MC energy

- Proto Algorithm has more accurate reconstruction energy

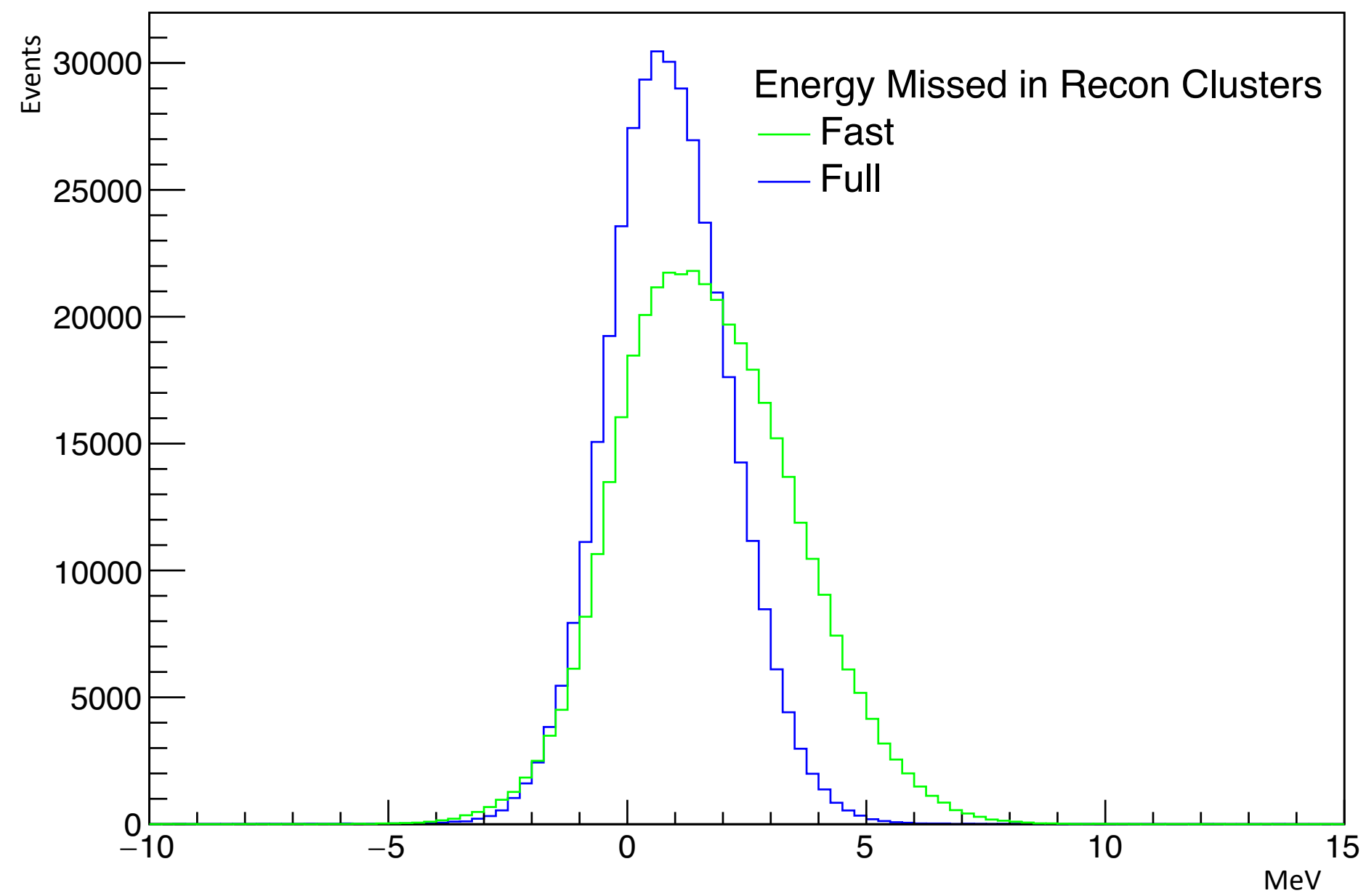

Fast:

Mean: 1.7

$\sigma: 1.81$

Full:

Mean: 0.87

$\sigma: 1.26$ 


\section{CE Only}

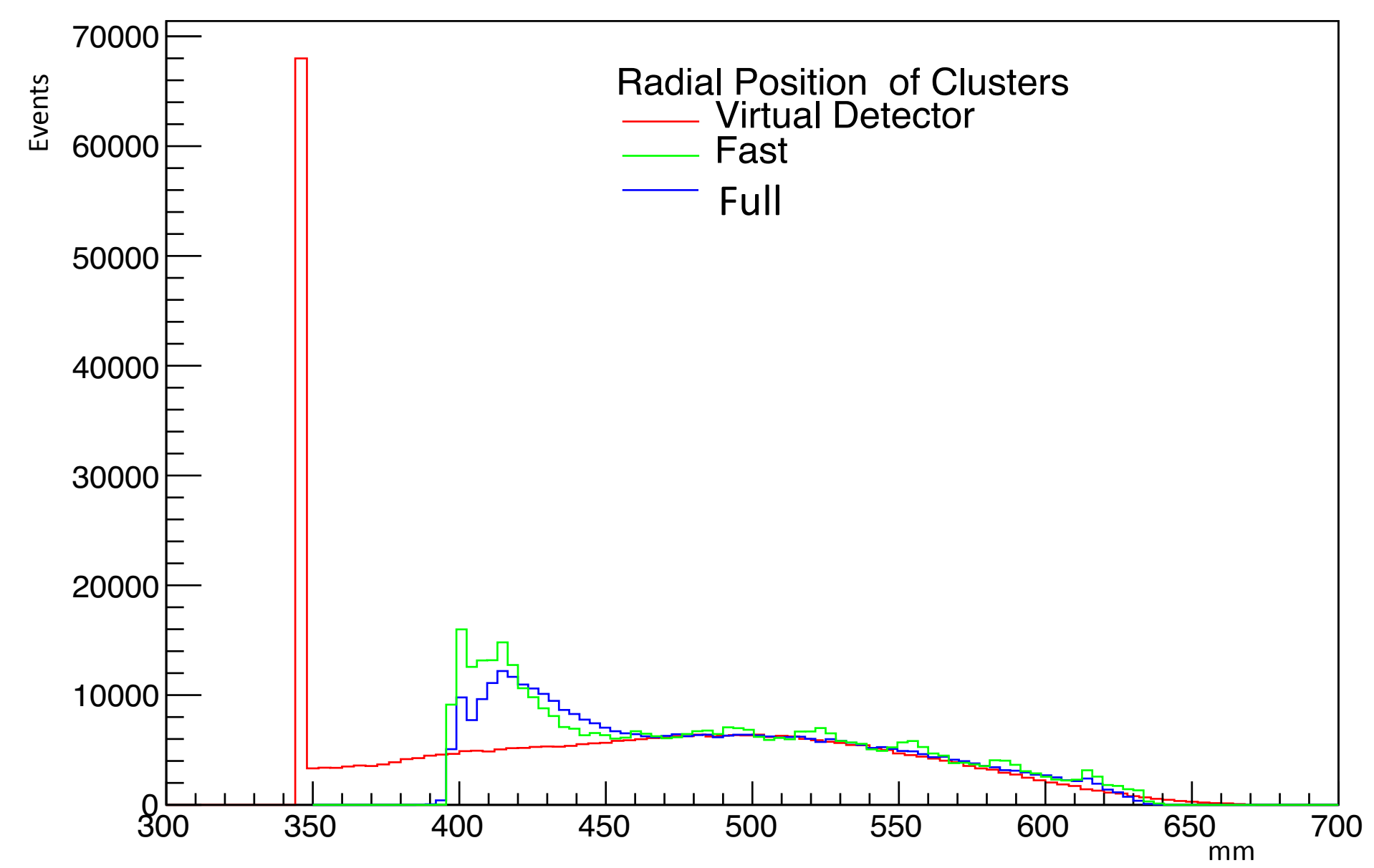




\section{1 -D}

Current list of histograms

Distribution of Cluster Energy versus Cluster Size Fast

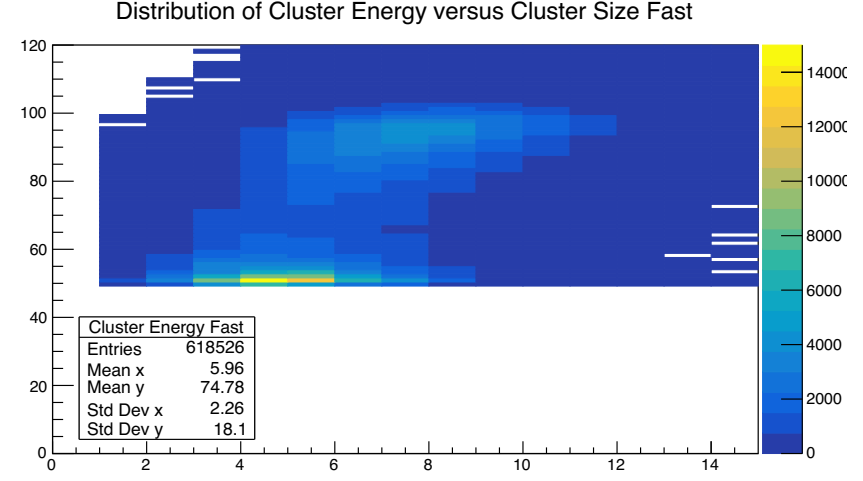

Distribution of Cluster Energy versus Cluster Size Proto

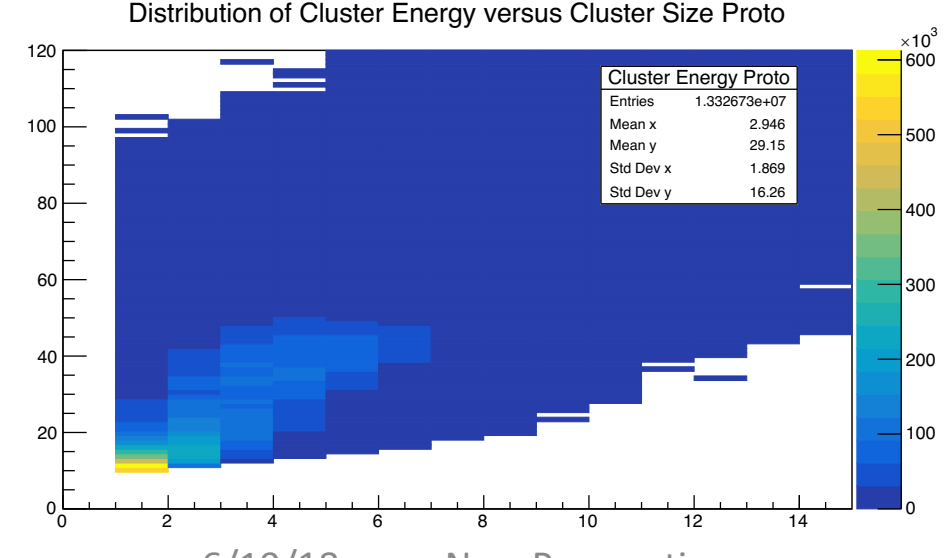

Timing of cluster

Energy of cluster

Energy of clusters over $50 \mathrm{MeV}$

Radial position of cluster

Energy of clusters outside $450 \mathrm{~mm}$

Angle of Cluster

Angle if cluster $>2$ crystals

Cluster Size

Seed Energy

MC truth w/recon energy

$\mathrm{MC}$ truth w/MC energy

Energy diff ( $\mathrm{MC}-$ Recon)

Ratio of Seed Energy to cluster energy

Highest Energy Cluster:

Recon Energy of cluster

Radial position of cluster

Angle of cluster

Size of cluster
2-D

Energy vs. Cluster Size

Energy vs. Angle

Energy vs. Time

Energy vs. Radial positon

Comp of MC and Recon crystal energy

Comp of MC and Recon Seed Energy

Comp or Ratio to Cluster Energy

Comp of Seed and Cluster Energy

\section{Virtual Detector}

Radial of True

Energy of True

Energy Missed by Fast

Energy Missed by proto 